\title{
VIEWING SOME ORDINARY DIFFERENTIAL EQUATIONS FROM THE ANGLE OF DERIVATIVE POLYNOMIALS
}

\author{
FENG QI \\ Institute of Mathematics, Henan Polytechnic University, Jiaozuo 454010, China; \\ College of Mathematics, Inner Mongolia University for Nationalities, Tongliao 028043, China; \\ Department of Mathematics, College of Science, Tianjin Polytechnic University, Tianjin 300387, China
}

\section{BAI-NI GUO}

School of Mathematics and Informatics, Henan Polytechnic University, Jiaozuo 454010, China

\begin{abstract}
In the paper, the authors view some ordinary differential equations and their solutions from the angle of (the generalized) derivative polynomials and simplify some known identities for the Bernoulli numbers and polynomials, the Frobenius-Euler polynomials, the Euler numbers and polynomials, in terms of the Stirling numbers of the first and second kinds.
\end{abstract}

\section{IntRoduction}

We recall some results obtained in two different and independent directions. We also recall below a concept in combinatorics about higher derivatives of functions.

1.1. The first direction. In [13, p. 1127 and 1131], it was obtained that

and

$$
\frac{1}{\left(1-e^{-t}\right)^{2}}=1+\frac{1}{e^{t}-1}-\left(\frac{1}{e^{t}-1}\right)^{\prime}
$$

$$
\frac{1}{\left(1-e^{-t}\right)^{3}}=1+\frac{1}{e^{t}-1}-\frac{3}{2}\left(\frac{1}{e^{t}-1}\right)^{\prime}+\frac{1}{2}\left(\frac{1}{e^{t}-1}\right)^{\prime \prime}
$$

In the preprint [17] and its formally published version [6], the above two identities were generalized inductively and recursively by the following eight identities:

$$
\left(\frac{1}{e^{t}-1}\right)^{(k)}=\sum_{m=1}^{k+1} \lambda_{k, m}\left(\frac{1}{e^{t}-1}\right)^{m},
$$

E-mail addresses: qifeng618@gmail.com, qifeng618@hotmail.com, qifeng618@qq.com, bai.ni.guo@gmail.com, bai.ni.guo@hotmail.com

2010 Mathematics Subject Classification. Primary 34A34, Secondary 11B68, 11B83, 26A24, $33 \mathrm{~B} 10$.

Key words and phrases. viewpoint; ordinary differential equation; solution; derivative polynomial; identity; Stirling numbers; Bernoulli number; Bernoulli polynomial; Frobenius-Euler polynomial.

This paper was typeset using $\mathcal{A M}_{\mathcal{M}}$-LATEX. 


$$
\begin{aligned}
\left(\frac{1}{1-e^{-t}}\right)^{(k)} & =\sum_{m=1}^{k+1} \mu_{k, m}\left(\frac{1}{1-e^{-t}}\right)^{m} \\
\left(\frac{1}{1-e^{-t}}\right)^{(k)} & =\sum_{m=1}^{k+1} \lambda_{k, m}\left(\frac{1}{e^{t}-1}\right)^{m} \\
\left(\frac{1}{e^{t}-1}\right)^{(k)} & =\sum_{m=1}^{k+1} \mu_{k, m}\left(\frac{1}{1-e^{-t}}\right)^{m}, \\
\left(\frac{1}{1-e^{-t}}\right)^{k} & =\sum_{m=1}^{k} a_{k, m-1}\left(\frac{1}{1-e^{-t}}\right)^{(m-1)}, \\
\left(\frac{1}{e^{t}-1}\right)^{k} & =\sum_{m=1}^{k} b_{k, m-1}\left(\frac{1}{e^{t}-1}\right)^{(m-1)} \\
\left(\frac{1}{1-e^{-t}}\right)^{k} & =1+\sum_{m=1}^{k} a_{k, m-1}\left(\frac{1}{e^{t}-1}\right)^{(m-1)} \\
\left(\frac{1}{e^{t}-1}\right)^{k} & =1+\sum_{m=1}^{k} b_{k, m-1}\left(\frac{1}{1-e^{-t}}\right)^{(m-1)}
\end{aligned}
$$

where $1 \leq m \leq k$, the quantities

$$
S(k, m)=\frac{1}{m !} \sum_{\ell=1}^{m}(-1)^{m-\ell}\left(\begin{array}{c}
m \\
\ell
\end{array}\right) \ell^{k}
$$

are the Stirling numbers of the second kind,

$$
\begin{gathered}
\lambda_{k, m}=(-1)^{k}(m-1) ! S(k+1, m), \quad \mu_{k, m}=(-1)^{m-1}(m-1) ! S(k+1, m), \\
a_{k, m-1}=(-1)^{m^{2}+1} M_{k-m+1}(k, m), \quad b_{k, m-1}=(-1)^{k-m} a_{k, m-1},
\end{gathered}
$$

and

$$
\begin{aligned}
& M_{j}(k, i)= \\
& \mid \begin{array}{ccccc}
\frac{1}{(i-1) !}\left(\begin{array}{c}
k \\
i
\end{array}\right) & S(i+1, i) & S(i+2, i) & \cdots & S(i+j-1, i) \\
\frac{1}{i !}\left(\begin{array}{c}
k \\
i+1
\end{array}\right) & S(i+1, i+1) & S(i+2, i+1) & \cdots & S(i+j-1, i+1) \\
\frac{1}{(i+1) !}\left(\begin{array}{c}
k \\
i+2
\end{array}\right) & 0 & S(i+2, i+2) & \cdots & S(i+j-1, i+2) \\
\vdots & \vdots & \vdots & \ddots & \vdots \\
\frac{1}{(i+j-2) !}\left(\begin{array}{c}
k \\
i+j-1
\end{array}\right) & 0 & 0 & \cdots & S(i+j-1, i+j-1)
\end{array}
\end{aligned}
$$

for $j \in \mathbb{N}$. In [6, Remark 5.1], it was pointed out that the four functions

$$
\frac{1}{e^{t}-1}, \quad \frac{1}{1-e^{-t}}, \quad \frac{1}{1-e^{-t}}, \quad \text { and } \quad \frac{1}{e^{t}-1}
$$

are respectively the unique solutions to the linear ordinary equations

$$
\sum_{i=1}^{k} a_{k, i-1} y^{(i-1)}=F_{n}(t) \text { and } \sum_{i=1}^{k}(-1)^{i-1} a_{k, i-1} y^{(i-1)}=G_{n}(t)
$$

for all $k \in \mathbb{N}$ and $n=1,2$, where

$$
F_{n}(t)=\left\{\begin{array}{ll}
\frac{1}{\left(1-e^{-t}\right)^{k}}-1, & n=1 ; \\
\frac{1}{\left(1-e^{-t}\right)^{k}}, & n=2
\end{array} \quad \text { and } \quad G_{n}(t)= \begin{cases}\frac{1}{\left(e^{t}-1\right)^{k}}-1, & n=1 \\
\frac{1}{\left(e^{t}-1\right)^{k}}, & n=2 .\end{cases}\right.
$$


In [6. Remark 5.2], it was concluded that

$$
\left(\begin{array}{c}
\frac{1}{e^{t}-1} \\
\left(\frac{1}{e^{t}-1}\right)^{\prime} \\
\left(\frac{1}{e^{t}-1}\right)^{\prime \prime} \\
\vdots \\
\left(\frac{1}{e^{t}-1}\right)^{(i-1)} \\
\left(\frac{1}{e^{t}-1}\right)^{(i)}
\end{array}\right)=\left(\begin{array}{ccccc}
\lambda_{0,1} & 0 & \cdots & 0 & 0 \\
\lambda_{1,1} & \lambda_{1,2} & \cdots & 0 & 0 \\
\lambda_{2,1} & \lambda_{2,2} & \cdots & 0 & 0 \\
\vdots & \vdots & \ddots & \vdots & \vdots \\
\lambda_{k-2,1} & \lambda_{k-2,2} & \cdots & \lambda_{k-2, k-1} & 0 \\
\lambda_{k-1,1} & \lambda_{k-1,2} & \cdots & \lambda_{k-1, k-1} & \lambda_{k-1, k}
\end{array}\right)\left(\begin{array}{c}
\frac{1}{e^{t}-1} \\
\frac{1}{\left(e^{t}-1\right)^{2}} \\
\frac{1}{\left(e^{t}-1\right)^{3}} \\
\vdots \\
\frac{1}{\left(e^{t}-1\right)^{i}} \\
\frac{1}{\left(e^{t}-1\right)^{i+1}}
\end{array}\right)
$$

and

$$
\left(\begin{array}{c}
\frac{1}{e^{t}-1} \\
\frac{1}{\left(e^{t}-1\right)^{2}} \\
\frac{1}{\left(e^{t}-1\right)^{3}} \\
\vdots \\
\frac{1}{\left(e^{t}-1\right)^{k}}
\end{array}\right)=(-1)^{(k-1) k / 2}\left(\begin{array}{ccccc}
a_{1,0} & 0 & 0 & \cdots & 0 \\
a_{2,0} & a_{2,1} & 0 & \cdots & 0 \\
a_{3,0} & a_{3,1} & a_{3,2} & \cdots & 0 \\
\vdots & \vdots & \vdots & \ddots & \vdots \\
a_{k, 0} & a_{k, 1} & a_{k, 2} & \cdots & a_{k, k-1}
\end{array}\right)\left(\begin{array}{c}
\frac{1}{e^{t}-1} \\
\left(\frac{1}{e^{t}-1}\right)^{\prime} \\
\left(\frac{1}{e^{t}-1}\right)^{\prime \prime} \\
\vdots \\
\left(\frac{1}{e^{t}-1}\right)^{(k-1)}
\end{array}\right) .
$$

All of the above results in [6, 17] were established by induction.

In [25, Theorems 3.1 and 3.2], by the Faà di Bruno formula and combinatorial techniques, the above identities in [6, 17] were generalized and unified as follows. For real numbers $\alpha, \lambda \in \mathbb{R}$,

(1) if $n \in \mathbb{N}$, then

$$
\left(\frac{1}{1-\lambda e^{\alpha t}}\right)^{(n)}=(-1)^{n} \alpha^{n} \sum_{k=1}^{n+1}(-1)^{k-1}(k-1) ! S(n+1, k)\left(\frac{1}{1-\lambda e^{\alpha t}}\right)^{k} ;
$$

(2) if $n \in \mathbb{N}$, then

$$
\left(\frac{1}{1-\lambda e^{\alpha t}}\right)^{n}=\frac{(-1)^{n-1}}{(n-1) !} \sum_{k=1}^{n} \frac{(-1)^{k-1}}{\alpha^{k-1}} s(n, k)\left(\frac{1}{1-\lambda e^{\alpha t}}\right)^{(k-1)}
$$

where $s(n, k)$ for $n \geq k \geq 1$ denote the Stirling numbers of the first kind.

In [4, Theorem 2.1], the above identities in [6, 17, 25] were inductively proved once again and were rewritten as follows. For real constants $\lambda \neq 0$ and $\alpha \neq 0$ and for $k \in \mathbb{N}$, when $\lambda>0$ and $t \neq-\frac{\ln \lambda}{\alpha}$ or when $\lambda<0$ and $t \in \mathbb{R}$, we have

$$
\frac{\mathrm{d}^{k}}{\mathrm{~d} t^{k}}\left(\frac{1}{\lambda e^{\alpha t}-1}\right)=(-1)^{k} \alpha^{k} \sum_{m=1}^{k+1}(m-1) ! S(k+1, m)\left(\frac{1}{\lambda e^{\alpha t}-1}\right)^{m}
$$

and

$$
\left(\frac{1}{\lambda e^{\alpha t}-1}\right)^{k}=\frac{1}{(k-1) !} \sum_{m=1}^{k} \frac{(-1)^{m-1}}{\alpha^{m-1}} s(k, m) \frac{\mathrm{d}^{m-1}}{\mathrm{~d} t^{m-1}}\left(\frac{1}{\lambda e^{\alpha t}-1}\right)
$$

1.2. The second direction. In [9, 10, it was obtained inductively and recursively that

(1) the function $F(t)=\frac{1}{u-e^{t}}$ is a solution of the nonlinear differential equation

$$
F^{n}(t)=\frac{n}{u^{n-1}} \sum_{k=0}^{n-1} \frac{1}{(k+1) !} \sum_{\ell_{1}+\ell_{2}+\cdots+\ell_{k+1}=n} \frac{1}{\ell_{1} \ell_{2} \cdots \ell_{k+1}} F^{(k)}(t),
$$

where $u \in \mathbb{C} \backslash\{1\}$ and $n \in \mathbb{N}$; 
(2) for $m \in \mathbb{N}$ and $n \geq 0$, we have

$$
H_{n}^{(m)}(u)=m\left(\frac{u-1}{u}\right)^{m-1} \sum_{k=0}^{m-1} \frac{1}{(k+1) !} \sum_{\ell_{1}+\ell_{2}+\cdots+\ell_{k+1}=m} \frac{H_{n+k}(u)}{\ell_{1} \ell_{2} \cdots \ell_{k+1}},
$$

where $H_{n}(u)$ are called the $n$th Frobenius-Euler numbers which can be generated by

$$
\frac{1-u}{e^{t}-u}=\sum_{n=0}^{\infty} H_{n}(u) \frac{t^{n}}{n !}, \quad u \neq 1
$$

(3) for $m \in \mathbb{N}$ and $n \geq 0$, we have

$$
\begin{aligned}
\sum_{\ell_{1}+\ell_{2}+\cdots+\ell_{m}=n}\left(\begin{array}{c}
n \\
\ell_{1}, \ell_{2}, \ldots, \ell_{m}
\end{array}\right) H_{\ell_{1}}(u) H_{\ell_{2}}(u) \cdots H_{\ell_{m}}(u) \\
=m\left(\frac{u-1}{u}\right)^{m-1} \sum_{k=0}^{m-1} \frac{1}{(k+1) !} \sum_{\ell_{1}+\ell_{2}+\cdots+\ell_{k+1}=m} \frac{H_{n+k}(u)}{\ell_{1} \ell_{2} \cdots \ell_{k+1}} ;
\end{aligned}
$$

(4) for $m \in \mathbb{N}$ and $n \geq 0$, we have

$$
\begin{aligned}
H_{n}^{(m)}(x \mid u)=m\left(\frac{u-1}{u}\right)^{m-1} & \sum_{k=0}^{m-1} \frac{1}{(k+1) !} \\
& \times \sum_{\ell_{1}+\ell_{2}+\cdots+\ell_{k+1}=m} \frac{1}{\ell_{1} \ell_{2} \cdots \ell_{k+1}} \sum_{q=0}^{n}\left(\begin{array}{c}
n \\
q
\end{array}\right) H_{q+k}(u) x^{n-q},
\end{aligned}
$$

where $H_{n}(x \mid u)$ are called the Frobenius-Euler polynomials which can be generated by

$$
\frac{1-u}{e^{t}-u} e^{x t}=\sum_{n=0}^{\infty} H_{n}(x \mid u) \frac{t^{n}}{n !}, \quad u \neq 1 .
$$

Influenced by the papers [9, 10, the authors in [7] derived inductively and recursively several formulas for the Bernoulli polynomials of the $r$ th order

$$
\left(\frac{t}{e^{t}-1}\right)^{r} e^{x t}=\sum_{n=0}^{\infty} B_{n}^{(r)}(x) \frac{t^{n}}{n !}
$$

in terms of the Bernoulli numbers $B_{n}=B_{n}^{(1)}(0)$.

In [22, Theorem 2.1], it was procured inductively and recursively that the function $F(t)=\frac{1}{e^{t}+1}$ is a solution of the nonlinear differential equation

$$
F^{n}(t)=n \sum_{k=0}^{n-1} \frac{1}{(k+1) !} \sum_{\ell_{1}+\ell_{2}+\cdots+\ell_{k+1}=n} \frac{1}{\ell_{1} \ell_{2} \cdots \ell_{k+1}} F^{(k)}(t), \quad n \in \mathbb{N} .
$$

By virtue of this, as did in [7, the authors of 22] presented several formulas for the Euler numbers and polynomials of the $r$ th order $E_{n}^{(r)}(0)$ and $E_{n}^{(r)}(x)$ defined by

$$
\left(\frac{2}{e^{t}+1}\right)^{r} e^{x t}=\sum_{n=0}^{\infty} E_{n}^{(r)}(x) \frac{t^{n}}{n !}
$$

in terms of the so-called Euler numbers $E_{n}=E_{n}^{(1)}(0)$ which are different from the classical and traditional Euler numbers $E_{n}=2^{n} E\left(\frac{1}{2}\right)$. 
In [8], it was acquired inductively and recursively that the function $F(t)=\frac{1}{\ln (1+t)}$ is a solution of the nonlinear differential equation

$$
F^{(n)}(t)=\frac{(-1)^{n}(n-1) !}{(1+t)^{n}} \sum_{j=2}^{n+1}(j-1) ! H_{n-1, j-2} F^{j}(t)
$$

for $n \in \mathbb{N}$, where

$$
H_{n, 0}=1, \quad H_{n, 1}=\sum_{k=1}^{n} \frac{1}{k}, \quad H_{0, j-1}=0, \quad H_{n, j}=\sum_{k=1}^{n} \frac{H_{k-1, j-1}}{k}
$$

for $2 \leq j \leq n$. Thereafter, the authors in [8] established an identity involving the higher order Bernoulli numbers of the second kind $b_{n}^{(k)}$ defined by

$$
\left[\frac{t}{\ln (1+t)}\right]^{k}=\sum_{n=0}^{\infty} b_{n}^{(k)} \frac{t^{n}}{n !}
$$

In [12, it was procured inductively and recursively that

(1) the function $F(t)=\frac{1}{(1+\lambda t)^{1 / \lambda}+1}$ is a solution of the nonlinear differential equation

$$
F^{(n)}(t)=\frac{(-1)^{n}}{(1+\lambda t)^{n}} \sum_{i=1}^{n+1}(-1)^{i-1} a_{i}(n, \lambda) F^{i}(t)
$$

for $n \in \mathbb{N}$, where

$$
\begin{aligned}
a_{i}(n, \lambda)=(i-1) ! \lambda^{n-i+1} \sum_{m_{i-1}=0}^{n-i+1} \sum_{m_{i-2}=0}^{n-m_{i-1}-i+1} \cdots \sum_{m_{1}=0}^{n-m_{i-1}-\cdots-m_{2}-i+1}\left(n-m_{i-1}\right. \\
\left.+\frac{i}{\lambda}\right)_{m_{i-1}}\left(n-m_{i-1}-m_{i-2}-1+\frac{i-1}{\lambda}\right)_{m_{i-2}} \cdots\left(n-m_{i-1}\right. \\
\left.-\cdots-m_{1}-i+2+\frac{2}{\lambda}\right)_{m_{1}}\left(\frac{1}{\lambda}\right)_{n-m_{i-1}-m_{i-2}-\cdots-m_{1}-i+1},
\end{aligned}
$$

(2) the function $F(t)=\frac{1}{(1+\lambda t)^{1 / \lambda}-1}$ is a solution of the nonlinear differential equation

$$
F^{(n)}(t)=\frac{(-1)^{n}}{(1+\lambda t)^{n}} \sum_{i=1}^{n+1} a_{i}(n, \lambda) F^{i}(t)
$$

for $n \in \mathbb{N}$.

Hereafter, the authors in [12] gave some new identities involving degenerate Euler numbers and polynomials.

For $x \in \mathbb{R}$, let

$$
\langle x\rangle_{n}= \begin{cases}x(x-1) \cdots(x-n+1), & n \geq 1 \\ 1, & n=0\end{cases}
$$

denotes the falling factorial of $x$. In [3, Theorem 2.1], it was presented inductively and recursively among other things that the function $F(t)=\frac{(1+t)^{x}}{2+t}$ is a solution of the linear differential equations

$$
F^{(n)}(t)=\left[\sum_{i=0}^{n} a_{i}(n, x) \frac{(2+t)^{i-n}}{(1+t)^{i}}\right] F(t),
$$

for $n \geq 0$, where $a_{0}(n, x)=(-1)^{n} n$ ! and

$$
a_{j}(n, x)=(-1)^{n-j}(n-j) !\langle x\rangle_{j}
$$




$$
\times \sum_{i_{j-1}=0}^{n-j} \sum_{i_{j-2}=0}^{n-j-i_{j-1}} \cdots \sum_{i_{1}=0}^{n-j-i_{j-1}-\cdots-i_{2}}\left(n-i_{j-1}-\cdots-i_{1}-j+1\right)
$$

for $1 \leq j \leq n$.

In [11], it was demonstrated inductively and recursively that

(1) the function $F(t)=\frac{1}{t+c}, t \neq-c$, is a solution of the nonlinear differential equation $F^{(n)}(t)=(-1)^{n} n ! F^{n+1}(t)$ for $n \in \mathbb{N}$,

(2) the function $G(t)=\frac{1}{e^{t}+1}$ is a solution of the nonlinear differential equation

$$
G^{(n)}(t)=(-1)^{n} \sum_{k=1}^{n+1}(-1)^{k-1} a_{n, k} G^{k}(t),
$$

where

$$
\begin{aligned}
a_{n, k}= & (k-1) ! \sum_{m_{1}=0}^{n-k+2} \sum_{m_{2}=0}^{n-k+2-m_{1}} \cdots \sum_{m_{k-2}=0}^{n-k+2-m_{1}-\cdots m_{k-3}} k^{m_{1}} \\
& \times(k-1)^{m_{2}} \cdots 3^{m_{k-2}}\left(2^{n-m_{1}-m_{2}-\cdots-m_{k-2}-k+3}-1\right) .
\end{aligned}
$$

Hereafter, the authors gave two identities for the Changhee polynomials of the $r$ th order $\mathrm{Ch}_{n}^{(r)}(x)$ defined by

$$
\left(\frac{2}{t+2}\right)^{r}(1+t)^{x}=\sum_{n=0}^{\infty} \mathrm{Ch}_{n}^{(r)}(x) \frac{t^{n}}{n !}
$$

1.3. Derivative polynomials. Suppose $f$ is a function whose derivative is a polynomial in $f$, that is, $f^{\prime}(x)=P(f(x))$ for some polynomial $P$. Then all the higher order derivatives of $f$ are also polynomials in $f$, so we have a sequence of polynomials $P_{n}$ defined by $f^{(n)}(x)=P_{n}(f(x))$ for $n \geq 0$. As usual, we call $P_{n}(u)$ the derivative polynomials of $f$. See [2, 24] and closely related references therein.

Now we more accurately introduce a new notion below. If there exists a sequence of polynomials $P_{n}(u)=\sum_{k=0}^{n} a_{k} u^{k}$ for $a_{k} \in \mathbb{C}$ and a sequence of functions $h_{n, k}(x)$ for $n, k \geq 0$ such that $f^{(n)}(x)=\sum_{k=0}^{n+q} a_{k} h_{n, k}(x) f^{k}(x)$ for $n, q \geq 0$, then we call $P_{n+q}(u)$ the generalized derivative polynomials of $f(x)$ with respect to $h_{n, k}(x)$.

\section{Alternative Viewpoints And DeRivative POlynomials}

Now we are in a position to discuss the above conclusions from alternative viewpoints and (the generalized) derivative polynomials.

2.1. Almost all the above linear or nonlinear ordinary differential equations and their solutions can be alternatively regarded as problems of (the generalized) derivative polynomials.

2.2. Taking $\lambda=-1$ and $\alpha=1$ in 1.1 and simplifying yield

$$
\frac{\mathrm{d}^{k}}{\mathrm{~d} t^{k}}\left(\frac{1}{e^{t}+1}\right)=(-1)^{k} \sum_{m=1}^{k+1}(-1)^{m-1}(m-1) ! S(k+1, m)\left(\frac{1}{e^{t}+1}\right)^{m} .
$$

Comparing this with 1.10 reveals that the coefficients $a_{n, k}$ in 1.11 is just equal to $(k-1) ! S(n+1, k)$. In other words,

$$
\begin{aligned}
S(n+1, k)= & \sum_{m_{1}=0}^{n-k+2} \sum_{m_{2}=0}^{n-k+2-m_{1}} \cdots \sum_{m_{k-2}=0}^{n-k+2-m_{1}-\cdots m_{k-3}} k^{m_{1}}(k-1)^{m_{2}} \cdots 3^{m_{k-2}} \\
& \times\left(2^{n-m_{1}-m_{2}-\cdots-m_{k-2}-k+3}-1\right) .
\end{aligned}
$$


We can also say that the generalized derivative polynomials of the function $\frac{1}{e^{t}+1}$ is

$$
P_{k+1}(x)=(-1)^{k} \sum_{m=1}^{k+1}(-1)^{m-1}(m-1) ! S(k+1, m) x^{m}
$$

with respect to $h_{k, m}(t) \equiv 1$ for $k \geq 1$ and $1 \leq m \leq k+1$. It is much straightforward and simple to see that the derivative polynomials of the function $\frac{1}{t+c}$ is $P_{n}(x)=$ $(-1)^{n} n ! x^{n+1}$. Consequently, we find an alternative viewpoint to examine the results in [11].

2.3. By Leibnlz's theorem for differentiation of a product, we obtain

$$
\begin{gathered}
\frac{\mathrm{d}^{n}}{\mathrm{~d} t^{n}}\left[\frac{(1+t)^{x}}{2+t}\right]=\sum_{k=0}^{n}\left(\begin{array}{l}
n \\
k
\end{array}\right) \frac{\mathrm{d}^{k}\left[(1+t)^{x}\right]}{\mathrm{d} t^{k}} \frac{\mathrm{d}^{n-k}}{\mathrm{~d} t^{n-k}}\left(\frac{1}{2+t}\right) \\
=\sum_{k=0}^{n}\left(\begin{array}{l}
n \\
k
\end{array}\right)\langle x\rangle_{k}(1+t)^{x-k} \frac{(-1)^{n-k}(n-k) !}{(2+t)^{n-k+1}} \\
=\sum_{k=0}^{n}\left(\begin{array}{l}
n \\
k
\end{array}\right)\langle x\rangle_{k} \frac{(-1)^{n-k}(n-k) !}{(1+t)^{k}(2+t)^{n-k}} \frac{(1+t)^{x}}{2+t}
\end{gathered}
$$

This implies that the coefficients $a_{j}(n, x)$ in 1.9$)$ can be simplified explicitly as

$$
a_{k}(n, x)=(-1)^{n-k}(n-k) !\left(\begin{array}{l}
n \\
k
\end{array}\right)\langle x\rangle_{k}=(-1)^{n-k} \frac{n !}{k !}\langle x\rangle_{k}, \quad 0 \leq k \leq n .
$$

Moreover, it follows that

$$
\left(\begin{array}{l}
n \\
k
\end{array}\right)=\sum_{i_{k-1}=0}^{n-k} \sum_{i_{k-2}=0}^{n-k-i_{k-1}} \cdots \sum_{i_{1}=0}^{n-k-i_{k-1}-\cdots-i_{2}}\left(n-i_{k-1}-\cdots-i_{1}-k+1\right) .
$$

In other words, the generalized derivative polynomials of the function $\frac{(1+t)^{x}}{2+t}$ is $P_{1+0}(u)=u$ with respect to

$$
h_{n, 1}(t)=\sum_{k=0}^{n}\left(\begin{array}{l}
n \\
k
\end{array}\right)\langle x\rangle_{k} \frac{(-1)^{n-k}(n-k) !}{(1+t)^{k}(2+t)^{n-k}}, \quad n \geq 0 .
$$

2.4. In combinatorial analysis, the Faà di Bruno formula plays an important role and can be described in terms of the Bell polynomials of the second kind

$$
\mathrm{B}_{n, k}\left(x_{1}, x_{2}, \ldots, x_{n-k+1}\right)=\sum_{\substack{1 \leq i \leq n, \ell_{i} \in\{0\} \cup \mathbb{N} \\ \sum_{i=1}^{n} i \ell_{i}=n \\ \sum_{i=1}^{n} \ell_{i}=k}} \frac{n !}{\prod_{i=1}^{n-k+1} \ell_{i} !} \prod_{i=1}^{n-k+1}\left(\frac{x_{i}}{i !}\right)^{\ell_{i}}
$$

for $n \geq k \geq 0$, see [1, p. 134 , Theorem A], by

$$
\frac{\mathrm{d}^{n}}{\mathrm{~d} t^{n}}[f \circ h(t)]=\sum_{k=0}^{n} f^{(k)}(h(t)) \mathrm{B}_{n, k}\left(h^{\prime}(t), h^{\prime \prime}(t), \ldots, h^{(n-k+1)}(t)\right)
$$

for $n \geq 0$, see [1, p. 139, Theorem C]. Replacing $f(u)$ and $u=h(t)$ in (2.1) respectively by $\frac{1}{u \pm 1}$ and $(1+\lambda t)^{1 / \lambda}$ yields

and

$$
f^{(k)}(u)=\frac{(-1)^{k} k !}{(u \pm 1)^{k+1}}, \quad h^{(k)}(t)=\left[\prod_{\ell=0}^{k-1}(1-\ell \lambda)\right](1+\lambda t)^{1 / \lambda-k},
$$

$$
\left[\frac{1}{(1+\lambda t)^{1 / \lambda} \pm 1}\right]^{(n)}=\sum_{k=0}^{n} \frac{(-1)^{k} k !}{\left[(1+\lambda t)^{1 / \lambda} \pm 1\right]^{k+1}} \mathrm{~B}_{n, k}\left((1+\lambda t)^{1 / \lambda-1}\right.
$$




$$
\begin{gathered}
\left.(1-\lambda)(1+\lambda t)^{1 / \lambda-k}, \ldots,\left[\prod_{\ell=0}^{n-k}(1-\ell \lambda)\right](1+\lambda t)^{1 / \lambda-n+k-1}\right) \\
=\frac{1}{(1+\lambda t)^{n}} \sum_{k=0}^{n} \frac{(-1)^{k} k !(1+\lambda t)^{k / \lambda}}{\left[(1+\lambda t)^{1 / \lambda} \pm 1\right]^{k+1}} \mathrm{~B}_{n, k}\left(1,1-\lambda, \ldots, \prod_{\ell=0}^{n-k}(1-\ell \lambda)\right),
\end{gathered}
$$

where we used in the above line the formula

$$
\mathrm{B}_{n, k}\left(a b x_{1}, a b^{2} x_{2}, \ldots, a b^{n-k+1} x_{n-k+1}\right)=a^{k} b^{n} \mathrm{~B}_{n, k}\left(x_{1}, x_{2}, \ldots, x_{n-k+1}\right)
$$

listed in [1, p. 135].

In [1, p. 133], it was listed that

$$
\frac{1}{k !}\left(\sum_{m=1}^{\infty} x_{m} \frac{t^{m}}{m !}\right)^{k}=\sum_{n=k}^{\infty} \mathrm{B}_{n, k}\left(x_{1}, x_{2}, \ldots, x_{n-k+1}\right) \frac{t^{n}}{n !}
$$

for $k \geq 0$. Therefore, we obtain that

$$
\begin{gathered}
\sum_{n=k}^{\infty} \mathrm{B}_{n, k}\left(1,1-\lambda, \ldots, \prod_{\ell=0}^{n-k}(1-\ell \lambda)\right) \frac{t^{n}}{n !}=\frac{1}{k !}\left(\sum_{m=1}^{\infty} \prod_{\ell=0}^{m-1}(1-\ell \lambda) \frac{t^{m}}{m !}\right)^{k} \\
=\frac{1}{k !}\left[(\lambda t+1)^{1 / \lambda}-1\right]^{k}=\frac{1}{k !} \sum_{\ell=0}^{k}(-1)^{k-\ell}\left(\begin{array}{c}
k \\
\ell
\end{array}\right)(\lambda t+1)^{\ell / \lambda}
\end{gathered}
$$

Accordingly, it follows that

$$
\begin{aligned}
& \mathrm{B}_{n, k}\left(1,1-\lambda,(1-\lambda)(1-2 \lambda), \ldots, \prod_{\ell=0}^{n-k}(1-\ell \lambda)\right) \\
= & \frac{1}{k !} \lim _{t \rightarrow 0} \sum_{\ell=0}^{k}(-1)^{k-\ell}\left(\begin{array}{l}
k \\
\ell
\end{array}\right)\left[(\lambda t+1)^{\ell / \lambda}\right]^{(n)} \\
= & \frac{1}{k !} \lim _{t \rightarrow 0} \sum_{\ell=0}^{k}(-1)^{k-\ell}\left(\begin{array}{l}
k \\
\ell
\end{array}\right) \prod_{q=0}^{n-1}(\ell-q \lambda)(1+\lambda t)^{\ell / \lambda-n} \\
= & \frac{(-1)^{k}}{k !} \sum_{\ell=0}^{k}(-1)^{\ell}\left(\begin{array}{l}
k \\
\ell
\end{array}\right) \prod_{q=0}^{n-1}(\ell-q \lambda) .
\end{aligned}
$$

In a word, we derive that

$$
\left[\frac{1}{(1+\lambda t)^{1 / \lambda} \pm 1}\right]^{(n)}=\sum_{k=0}^{n}\left[\sum_{\ell=0}^{k}(-1)^{\ell}\left(\begin{array}{l}
k \\
\ell
\end{array}\right) \prod_{q=0}^{n-1}(\ell-q \lambda)\right] \frac{(1+\lambda t)^{k / \lambda-n}}{\left[(1+\lambda t)^{1 / \lambda} \pm 1\right]^{k+1}}
$$

for $n \geq 0$. This means that the functions $\frac{1}{(1+\lambda t)^{1 / \lambda} \pm 1}$ have the same generalized derivative polynomials

$$
P_{n+1}(u)=\sum_{k=1}^{n+1}\left[\sum_{\ell=0}^{k-1}(-1)^{\ell}\left(\begin{array}{c}
k-1 \\
\ell
\end{array}\right) \prod_{q=0}^{n-1}(\ell-q \lambda)\right] u^{k}
$$

with respect to $h_{n, k}(t)=(1+\lambda t)^{(k-1) / \lambda-n}$ for $n \geq 0$ and $1 \leq k \leq n+1$.

By the way, we note that the form of the equation 2.3 is different from the one in 1.6 and 1.8 . The coefficients in the brackets on the right-hand side of the equation 2.3 are more nice, more explicit, easier to compute than the coefficients $a_{i}(n, \lambda)$ defined by (1.7) in (1.6) and $(1.8)$. 
2.5. In [14, 18, it was obtained that

$$
\left[\frac{1}{\ln (1+t)}\right]^{(m)}=\frac{1}{(1+t)^{m}} \sum_{i=0}^{m}(-1)^{i} i ! \frac{s(m, i)}{[\ln (1+t)]^{i+1}}, \quad m \geq 0
$$

and

$$
\left(\frac{1}{\ln x}\right)^{(n)}=\frac{(-1)^{n}}{x^{n}} \sum_{i=2}^{n+1} \frac{a_{n, i}}{(\ln x)^{i}}, \quad n \in \mathbb{N},
$$

where $a_{n, 2}=(n-1)$ ! and

$$
a_{n, i}=(i-1) !(n-1) ! \sum_{\ell_{1}=1}^{n-1} \frac{1}{\ell_{1}} \sum_{\ell_{2}=1}^{\ell_{1}-1} \frac{1}{\ell_{2}} \cdots \sum_{\ell_{i-3}=1}^{\ell_{i-4}-1} \frac{1}{\ell_{i-3}} \sum_{\ell_{i-2}=1}^{\ell_{i-3}-1} \frac{1}{\ell_{i-2}}
$$

for $n+1 \geq i \geq 3$. Comparing this with 1.5 and rearranging lead to

$$
H_{n-1, i-1}=\sum_{\ell_{1}=1}^{n-1} \frac{1}{\ell_{1}} \sum_{\ell_{2}=1}^{\ell_{1}-1} \frac{1}{\ell_{2}} \cdots \sum_{\ell_{i-3}=1}^{\ell_{i-4}-1} \frac{1}{\ell_{i-3}} \sum_{\ell_{i-2}=1}^{\ell_{i-3}-1} \frac{1}{\ell_{i-2}}=(-1)^{n+i} \frac{s(n, i)}{(n-1) !}
$$

for $3 \leq i \leq n$. This connects 1.5 with the Stirling numbers of the first kind $s(n, i)$ and the generalized derivative polynomials: the generalized derivative polynomials of $\frac{1}{\ln (1+t)}$ is

$$
P_{m+1}(u)=\sum_{i=1}^{m+1}(-1)^{i-1}(i-1) ! s(m, i-1) u^{i}
$$

with respect to $h_{m, i} \equiv \frac{1}{(1+t)^{m}}$ for $m \geq 0$. Hence, we are viewing the paper [8] from a different angle.

2.6. Letting $\lambda=-1$ and $\alpha=1$ in 1.2 results in

$$
\left(\frac{1}{e^{t}+1}\right)^{k}=\frac{1}{(k-1) !} \sum_{m=1}^{k}(-1)^{m+k} s(k, m) \frac{\mathrm{d}^{m-1}}{\mathrm{~d} t^{m-1}}\left(\frac{1}{e^{t}+1}\right) .
$$

Comparing this with 1.4 gives

$$
s(n, k+1)=(-1)^{n+k+1} \frac{n !}{(k+1) !} \sum_{\sum_{q=1}^{k+1} \ell_{q}=n} \prod_{q=1}^{k+1} \frac{1}{\ell_{q}}, \quad n \in \mathbb{N} .
$$

As a result, Theorem 2.1 in 22 can be simplified as 2.4 and has something to do with the Stirling numbers of the first kind $s(n, k)$.

2.7. Setting $\lambda=\frac{1}{u}$ and $\alpha=1$ in 1.2 produces

$$
\left(\frac{1}{u-e^{t}}\right)^{k}=\frac{(-1)^{k-1}}{(k-1) !} \frac{1}{u^{k-1}} \sum_{m=0}^{k-1}(-1)^{m} s(k, m+1) \frac{\mathrm{d}^{m}}{\mathrm{~d} t^{m}}\left(\frac{1}{u-e^{t}}\right)
$$

for $k \in \mathbb{N}$. Comparing this with 1.3 recovers the formula 2.5 which is an alternative expression for the Stirling numbers of the first kind $s(n, k)$. Therefore, all the above-mentioned results in 9, 10, can be restated simply in terms of the Stirling numbers of the first kind $s(n, k)$.

2.8. In [4, 6, 25], some formulas for computing the Bernoulli numbers, the Euler polynomials, the Apostol-Bernoulli numbers, the Eulerian polynomials, and the Fubini numbers in terms of the Stirling numbers of the second kind were established. These formulas are more concise, simpler, more meaningful than those in [7, 8, 9 , 10, 11, 22, as showed above. Due to limitation on the length of the paper, we will not elaborate in further detail. 
2.9. In 5, 15, 20, 21, 23, 24, there are more information and new conclusions about (the generalized) derivative polynomials, explicit formulas for the Bernoulli numbers and polynomials, for the Euler numbers and polynomials, for higher derivatives of some elementary functions, properties of the functions $\frac{ \pm 1}{e^{ \pm t}-1}$, and the like. Due to limitation on the length of the paper, we will not elaborate in further detail yet.

2.10. It should be common knowledge that mathematicians should try to represent, explain, or interpret a new mathematical quantities in terms of some known and popular quantities in mathematics. Once a new mathematical quantity were established connections or relations with some famous or important quantities, it would be more meaningful in mathematics.

\section{Remarks}

Finally we would like to give several remarks.

Remark 1. From the derivation of the equation $[2.3)$, we can conclude that

$$
\begin{aligned}
\mathrm{B}_{n, k}\left(1,1-\lambda,(1-\lambda)(1-2 \lambda), \ldots, \prod_{\ell=0}^{n-k}(1-\ell \lambda)\right) \\
=\frac{(-1)^{k}}{k !} \sum_{\ell=0}^{k}(-1)^{\ell}\left(\begin{array}{c}
k \\
\ell
\end{array}\right) \prod_{q=0}^{n-1}(\ell-q \lambda)
\end{aligned}
$$

for $n \geq k \geq 0$. This formula can be used to derive many known and new special values of the Bell polynomials of the second kind $\mathrm{B}_{n, k}$ such as, when taking $\lambda=$ $0,-1,2, \frac{1}{2},-2,-\frac{1}{2}$ in 3.1 respectively,

$$
\begin{gathered}
\mathrm{B}_{n, k}(1,1,1, \ldots, 1)=\frac{(-1)^{k}}{k !} \sum_{\ell=0}^{k}(-1)^{\ell}\left(\begin{array}{l}
k \\
\ell
\end{array}\right) \ell^{n}=S(n, k), \\
\mathrm{B}_{n, k}(1 !, 2 !, 3 !, \ldots,(n-k+1) !)=\frac{(-1)^{k}}{k !} \sum_{\ell=0}^{k}(-1)^{\ell}\left(\begin{array}{l}
k \\
\ell
\end{array}\right) \prod_{q=0}^{n-1}(\ell+q) \\
=\left(\begin{array}{l}
n \\
k
\end{array}\right)\left(\begin{array}{l}
n-1 \\
k-1
\end{array}\right)(n-k) ! \\
=L(n, k), \\
\mathrm{B}_{n, k}((-1) ! !, 1 ! !, 3 ! !, \ldots,(2(n-k)-1) ! !)=\frac{(-1)^{k}}{k !} \sum_{\ell=0}^{k}(-1)^{\ell}\left(\begin{array}{l}
k \\
\ell
\end{array}\right) \prod_{q=0}^{n-1}(\ell-2 q), \\
\mathrm{B}_{n, k}\left(1, \frac{1}{2}, 0, \ldots, 0\right)=\frac{(-1)^{k}}{k !} \sum_{\ell=0}^{k}(-1)^{\ell}\left(\begin{array}{c}
k \\
\ell
\end{array}\right) \prod_{q=0}^{n-1}\left(\ell-\frac{q}{2}\right) \\
=\frac{(n-k) !}{4^{n-k}\left(\begin{array}{l}
n \\
k
\end{array}\right)\left(\begin{array}{c}
k \\
n-k
\end{array}\right)} \\
\mathrm{B}_{n, k}(1 ! !, 3 ! !, 5 ! !, \ldots,(2(n-k)+1) ! !)=\frac{(-1)^{k}}{k !} \sum_{\ell=0}^{k}(-1)^{\ell}\left(\begin{array}{l}
k \\
\ell
\end{array}\right) \prod_{q=0}^{n-1}(\ell+2 q),
\end{gathered}
$$

and

$$
\mathrm{B}_{n, k}\left(1, \frac{3}{2}, 3, \frac{15}{2} \ldots, \frac{(n-k+2) !}{2^{n-k+1}}\right)=\frac{(-1)^{k}}{k !} \sum_{\ell=0}^{k}(-1)^{\ell}\left(\begin{array}{c}
k \\
\ell
\end{array}\right) \prod_{q=0}^{n-1}\left(\ell+\frac{q}{2}\right)
$$

for $n \geq k \geq 0$, where $L(n, k)$ for $n \geq k \geq 0$ denote the Lah numbers. The identities $(3.2$ and $(3.3)$ can be found in [1, p. 135]. The identity (3.3) can also 
be found in [16, Theorem 1]. The identity (3.4) recovers the main results [20, Theorems 1 and 2]. By virtue of the formula (2.2), the identity (3.5) is a special case of

$$
\mathrm{B}_{n, k}(x, 1,0, \ldots, 0)=\frac{(n-k) !}{2^{n-k}}\left(\begin{array}{l}
n \\
k
\end{array}\right)\left(\begin{array}{c}
k \\
n-k
\end{array}\right) x^{2 k-n}
$$

which was established alternatively in [5, Theorem 4.1], [20, Eq. (2.8)], and [21, Section 3]. The identities (3.6) and (3.7) are, to the best of our knowledge, new.

Remark 2. By the way, the formulas (3.4) and (3.5) in [18, p. 325, Corollary 3.1] should be slightly corrected as

$$
\begin{aligned}
& {\left[\frac{x}{\ln (1+x)}\right]^{(i)}=\frac{(-1)^{i}}{(1+x)^{i}} \sum_{k=2}^{i+1} \frac{x a_{i, k}-i(1+x) a_{i-1, k}}{[\ln (1+x)]^{k}}, \quad i \geq 2,} \\
& {\left[\frac{x}{\ln (1+x)}\right]^{(i)}=\frac{1}{(1+x)^{i}} \sum_{k=1}^{i} \frac{(-1)^{k} k ![x s(i, k)+i(1+x) s(i-1, k)]}{[\ln (1+x)]^{k+1}}, \quad i \geq 2,}
\end{aligned}
$$

or

$$
\left[\frac{x}{\ln (1+x)}\right]^{(i)}=\frac{1}{(1+x)^{i}} \sum_{k=0}^{i} \frac{(-1)^{k} k ![x s(i, k)+i(1+x) s(i-1, k)]}{[\ln (1+x)]^{k+1}}, \quad i \geq 1
$$

where $a_{n, 2}=(n-1)$ ! for $n \in \mathbb{N}$,

$$
a_{n, i}=(i-1) !(n-1) ! \sum_{\ell_{1}=1}^{n-1} \frac{1}{\ell_{1}} \sum_{\ell_{2}=1}^{\ell_{1}-1} \frac{1}{\ell_{2}} \cdots \sum_{\ell_{i-3}=1}^{\ell_{i-4}-1} \frac{1}{\ell_{i-3}} \sum_{\ell_{i-2}=1}^{\ell_{i-3}-1} \frac{1}{\ell_{i-2}}
$$

for $n+1 \geq i \geq 3$, and

$$
a_{n, i}=(-1)^{n+i-1}(i-1) ! s(n, i-1)
$$

for $2 \leq i \leq n+1$.

Remark 3. This paper is a slightly revised and extended version of the preprint [19].

Acknowledgments. The first author thanks Professors Taekyun Kim and LeeChae Jang at Kwangwoon University and Konkuk University in South Korea for their academic visit and nice lectures between 22-26 February 2016 at Tianjin Polytechnic University in China and for their recommending the papers [11, 12.

\section{REFERENCES}

[1] L. Comtet, Advanced Combinatorics: The Art of Finite and Infinite Expansions, Revised and Enlarged Edition, D. Reidel Publishing Co., Dordrecht and Boston, 1974.

[2] D. Cvijović, Derivative polynomials and closed-form higher derivative formulae, Appl. Math. Comput. 215 (2009), no. 8, 3002-3006; Available online at http://dx.doi.org/10.1016/j. amc. 2009.09.047

[3] D. V. Dolgy, D. S. Kim, T. Kim, J.-J. Seo, Differential equations for Changhee polynomials and their applications, arXiv preprint (2016), available online at http://arxiv.org/abs/ 1602.08659

[4] B.-N. Guo and F. Qi, Explicit formulae for computing Euler polynomials in terms of Stirling numbers of the second kind, J. Comput. Appl. Math. 272 (2014), 251-257; Available online at http://dx.doi.org/10.1016/j.cam.2014.05.018

[5] B.-N. Guo and F. Qi, Explicit formulas for special values of the Bell polynomials of the second kind and the Euler numbers, ResearchGate Technical Report (2015), available online at http://dx.doi.org/10.13140/2.1.3794.8808

[6] B.-N. Guo and F. Qi, Some identities and an explicit formula for Bernoulli and Stirling numbers, J. Comput. Appl. Math. 255 (2014), 568-579; Available online at http://dx.doi. org $/ 10.1016 / j$.cam.2013.06.020

[7] D. Kang, J. Jeong, S.-J. Lee, and S.-H. Rim, A note on the Bernoulli polynomials arising from a non-linear differential equation, Proc. Jangjeon Math. Soc. 16 (2013), no. 1, 37-43. 
[8] D. S. Kim and T. Kim, Some identities for Bernoulli numbers of the second kind arising from a non-linear differential equation, Bull. Korean Math. Soc. 52 (2015), no. 6, 2001-2010; Available online at http://dx.doi.org/10.4134/BKMS.2015.52.6.2001

[9] T. Kim, Identities involving Frobenius-Euler polynomials arising from non-linear differential equations, J. Number Theory 132 (2012), no. 12, 2854-2865; Available online at http://dx. doi.org/10.1016/j.jnt.2012.05.033

[10] T. Kim, Corrigendum to "Identities involving Frobenius-Euler polynomials arising from nonlinear differential equations" [J. Number Theory 132 (12) (2012) 2854-2865], J. Number Theory 133 (2013), no. 2, 822-824; Available online at http://dx.doi.org/10.1016/j.jnt. 2012.08 .002

[11] T. Kim and D. S. Kim, A note on nonlinear Changhee differential equations, Russ. J. Math. Phys. 23 (2016), no. 1, 88-92; Available online at http://dx.doi.org/10.1134/ S1061920816010064.

[12] T. Kim and D. S. Kim, Identities involving degenerate Euler numbers and polynomials arising from non-linear differential equations, J. Nonlinear Sci. Appl. 9 (2016), no. 5, 2086-2098.

[13] S. Koumandos, On Ruijsenaars' asymptotic expansion of the logarithm of the double gamma function, J. Math. Anal. Appl. 341 (2008), 1125-1132; Available online at http://dx.doi. org $/ 10.1016 / j \cdot j$ jmaa.2007.11.021

[14] H.-M. Liu, S.-H. Qi, and S.-Y. Ding, Some recurrence relations for Cauchy numbers of the first kind, J. Integer Seq. 13 (2010), Article 10.3.8, 7 pages.

[15] F. Qi, Derivatives of tangent function and tangent numbers, Appl. Math. Comput. 268 (2015), 844-858; Available online at http://dx.doi.org/10.1016/j.amc.2015.06.123

[16] F. Qi, Diagonal recurrence relations for the Stirling numbers of the first kind, Contrib. Discrete Math. 11 (2016), no. 1, 22-30; Available online at http://hdl.handle.net/10515/ sy5wh2dx6 and http://dx.doi.org/10515/sy5wh2dx6

[17] F. Qi, Eight interesting identities involving the exponential function, derivatives, and Stirling numbers of the second kind, arXiv preprint (2012), available online at http://arxiv.org/abs/ 1202.2006

[18] F. Qi, Explicit formulas for computing Bernoulli numbers of the second kind and Stirling numbers of the first kind, Filomat 28 (2014), no. 2, 319-327; Available online at http: //dx.doi.org/10.2298/FIL14023190

[19] F. Qi and B.-N. Guo, Viewing some nonlinear ODEs and their solutions from the angle of derivative polynomials, ResearchGate Working Paper (2016), available online at http: $/ / \mathrm{dx} . \mathrm{doi} . \mathrm{org} / 10.13140 / \mathrm{RG} .2 .1 .4593 .1285$

[20] F. Qi, X.-T. Shi, F.-F. Liu, and D. V. Kruchinin, Several formulas for special values of the Bell polynomials of the second kind and applications, J. Appl. Anal. Comput. 7 (2017), in press; ResearchGate Technical Report (2015), available online at http://dx.doi.org/10. 13140/RG.2.1.3230.1927

[21] F. Qi and M.-M. Zheng, Explicit expressions for a family of the Bell polynomials and applications, Appl. Math. Comput. 258 (2015), 597-607; Available online at http://dx.doi.org/ 10.1016/j.amc.2015.02.027

[22] S.-H. Rim, J. Jeong, and J.-W. Park, Some identities involving Euler polynomials arising from a non-linear differential equation. Kyungpook Math. J. 53 (2013), no. 4, 553-563; Available online at http://dx.doi.org/10.5666/KMJ.2013.53.4.553

[23] C.-F. Wei and B.-N. Guo, Complete monotonicity of functions connected with the exponential function and derivatives, Abstr. Appl. Anal. 2014 (2014), Article ID 851213, 5 pages; Available online at http://dx.doi.org/10.1155/2014/851213

[24] A.-M. Xu and G.-D. Cen, Closed formulas for computing higher-order derivatives of functions involving exponential functions, Appl. Math. Comput. 270 (2015), 136-141; Available online at http://dx.doi.org/10.1016/j.amc.2015.08.051

[25] A.-M. Xu and Z.-D. Cen, Some identities involving exponential functions and Stirling numbers and applications, J. Comput. Appl. Math. 260 (2014), 201-207; Available online at http://dx.doi.org/10.1016/j.cam.2013.09.077

$U R L:$ https : //qifeng618.wordpress.com

$U R L:$ http://www.researchgate.net/profile/Bai-Ni_Guo/

(C) 2016 by the authors; licensee Preprints, Basel, Switzerland. This article is an open access article distributed under the terms and conditions of the Creative Commons by Attribution (CC-BY) license (http://creativecommons.org/licenses/by/4.0/). 